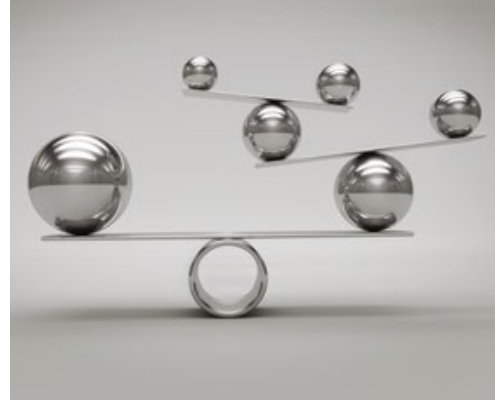

CPD

Jill Thistlethwaite, Lynn Weeks

\section{Background}

In Australia, the Therapeutic Goods Administration (TGA) approves new medicines that general practitioners (GPs) may prescribe. Medicines listed under the Pharmaceutical Benefits Scheme (PBS) have undergone a rigorous independent evaluation in relation to comparative efficacy, safety and effectiveness against medicines already on the market. However, GPs need to appraise the medicines they prescribe for individual patients, taking a number of factors into consideration.

\section{Objectives}

The aim of this article is to assist GPs' decision making when prescribing new medicines, and provide information about the most reliable sources of unbiased information to help them evaluate the risks and benefits in partnership with their patients.

\section{Discussion}

GPs require accurate summaries of a medicine's properties, efficacy and side-effect profile from trusted sources such as the Australian Medicines Handbook, Therapeutic Guidelines, NPS MedicineWise, Australian Prescriber or the TGA. Information about a new medicine needs to be interpreted so that patients may also make informed choices.

\title{
Evaluating and prescribing new medicines in general practice
}

IN THE DECADE up to and including 2011, the Therapeutic Goods Administration (TGA) approved 795 new medicines for 93 different conditions in Australia. ${ }^{1}$ The top three indications were for cancer, cardiovascular diseases and vaccination. Forty-two new chemical entities were registered in 2016, including some for the treatment of hyperlipidaemia and hepatitis $\mathrm{C}$ infection. ${ }^{2}$ While many of these medicines are for highly specialised usage, some will be appropriate for patients managed in general practice. General practitioners (GPs) may initiate prescriptions of new medicines, or they may be asked to continue prescriptions by other health professionals. This article discusses the information available to assist GPs in making decisions about prescribing medicines with which they are not familiar, including advice about reliable sources of unbiased information, to help evaluate risks and benefits in partnership with patients.

\section{The regulation of new medicines}

In Australia, new medicines undergo a rigorous process by the TGA to evaluate their efficacy and safety. This may include the TGA requesting a pharmaceutical company's evidence about how new medicines are likely to behave specifically in Australia's diverse and multi-ethnic population. ${ }^{3}$ Doctors may therefore think it safe to assume that registered medicines are therapeutically effective and have a good safety profile. However, some drugs are withdrawn within a short time of being marketed. This is usually due to a greater incidence of adverse events than expected when used by a larger number of patients and a more diverse population than the patients in clinical trials. Examples of medicines withdrawn in Australia as a result of adverse events include rofecoxib in 2004 and sibutramine in $2010 .{ }^{4}$ It is important to note that the TGA does not consider the cost-effectiveness of medicines, nor does it recommend one product over another similar one on the market. ${ }^{5}$

Medicines listed on the Pharmaceutical Benefits Scheme (PBS) are deemed 'necessary for Australians' and cost effective in terms of price and outcomes ${ }^{6}$ following an independent evaluation by the Pharmaceutical Benefits Advisory Committee (PBAC) in relation to comparative efficacy, safety and costeffectiveness against medicines already on the market. They are restricted to specific therapeutic indications tested in clinical trials and, preferably, in head-tohead studies with other medicines rather than placebo. Medicines may be available for some time before they are included on the PBS and, even when listed, a prescriber must consider the needs and context of an individual patient in relation to a specific medicine.

\section{Evaluating medicines}

Prescribers take responsibility for appraising the medicines they choose for individual patients, taking into account past history, current medications, allergies and preferences, as well as ensuring that healthcare resources are used wisely 
for the benefit of patients. ${ }^{7}$ GPs need to consider not only what is known about a drug but also what is not yet known. Gold standard randomised clinical trials (RCTs) used during the initial testing of new drugs are conducted with a small number of highly selected participants over short periods of time. Research participants are unlikely to be identical to patients in the community who have multimorbidities and who are already taking several medicines. Outcomes of trials may not be the outcomes that GPs are interested in for their patients or that patients most value. For example, RCTs may look at lowering blood glucose or cholesterol as end-points, rather than clinical events such as myocardial infarctions or strokes. In addition, not all RCT data are published or easily accessible. ${ }^{8}$ While there is a global minimum set of recommendations for reporting RCTs to improve transparency and aid in appraisal and interpretation, ${ }^{9}$ inconclusive trials or those showing negative outcomes may not have been published. ${ }^{8}$ Larger clinical trials are powered in terms of patient numbers to show effectiveness but may not have sufficient numbers to identify uncommon adverse events.

There is a move towards looking at patient-reported outcomes measures (PROMs), which are more relevant to patients and indicate how treatments affect their day-to-day quality of life and functioning. Research in this area is providing evidence that PROMs help determine the relative effectiveness of different treatment options and interventions while enhancing patientclinician interactions. ${ }^{10}$ Evaluations of new medicines are likely to incorporate PROMs in the future.

\section{What to know before prescribing a new medicine}

Even if a new medicine is initially prescribed by another health professional, for example during a hospital admission or specialist consultation, there is important information about a medicine that GPs should know ${ }^{11}$ (Boxes 1, 2). GPs receive updates to their knowledge on a daily basis and require easy access to relevant and succinct information. Few will want, or have the time, to read in detail about the outcomes of RCTs. In our experience, GPs want accurate summaries of a medicine's properties, efficacy and side-effect profile from a trusted source on which to base a decision. A medicine's product information (PI), available in general practice clinical software, is a useful starting point, but some very new drugs may not have had a PI lodged. PIs are not regularly updated and do not present information comparing different medicines or best-practice recommendations for management. New medicines have limited evidence to appraise apart from TGA evaluations and this needs to be taken into account in decision making. Sources of independent information about medicines in Australia are given in Box 3. Other useful resources globally are listed in Australian Prescriber. ${ }^{12}$

A 'new' medicine is not always completely new: it may be a member of an existing class (eg a statin or inhaled steroid) or administered via a new delivery system (eg a spray rather than tablet), or there may be a new indication for an existing medicine with considerable evidence already available. Consideration should be given to whether there is any benefit of a new medicine for a given patient, compared with what is more familiar. Some medicines will have been available in other countries prior to regulation in Australia and have post-marketing surveillance data to appraise in relation to the Australian context. However, for some medicines, there may not be information about use in combinations with other medicines, or in the elderly, in children or during pregnancy.

Prescribing a new medicine is an uncertain process. GPs' concerns about the safety and efficacy of new medicines affect their uptake. ${ }^{13}$ The safety profile of a new medicine may not be fully understood immediately following an RCT. Only as the drug is used more widely will rarer adverse events become more obvious: exposure to at least 30,000 people is required for reliable detection of an adverse drug reaction that occurs in one in 10,000 patients. ${ }^{14}$ It is extremely important to report all suspected adverse events to the Advisory Committee on Medicines (ACM) via the blue card scheme available at www. tga.gov.au/form/blue-card-adversereaction-reporting-form ${ }^{15}$ and check the TGA website, which lists medicine safety reviews regularly. ${ }^{16}$ Adverse events should be recorded in a patient's medical record.

\section{Shared decision making about treatment}

Whenever a patient takes a medicine that is new for them, it is in the nature of an experiment: neither the patient nor the

\section{Box 1. Questions to ask before prescribing a new medicine"}

- What is new or different about this medicine?

- Is it a 'me too' drug or something innovative?

- Is there good quality evidence that it is more effective than existing medication?

- Are there any studies comparing it to existing medicines?

- Is the evidence based on RCTs with sufficient participants?

- Are the studies relevant to my patient/ population?

- Are there any longer term studies with patient-relevant outcomes?

- Are there good safety data?

- What are the incidence and severity of side effects?

- Are long-term safety data available?

- Which patients are at most risk of side effects?

- Which patients should not receive this medicine?

- Do the benefits from this medicine outweigh the risks involved?

- Are there some patients who may particularly benefit from this medicine?

- What is its cost?

- Is it more expensive that existing therapy and if so is this justified?

- Is it PBS-listed and if so what is the indication?

- Is it acceptable to patients?

- In balancing the possible benefits and risks of harm?

- In relation to formulation, route of administrations and dose frequency?

- Is monitoring required?

$R C T s$, randomised clinical trials;

PBS, Pharmaceutical Benefits Scheme 
prescriber can predict exactly what will happen. GPs prescribing medicines with which they are familiar have a personal database of knowledge, experience and outcomes on which to base their prescribing choices and the information they share with patients. The reinforced and internalised personal guidelines on which GPs rely in practice have been termed 'mindlines'. ${ }^{17}$ These mindlines

\section{Box 2. Example: Empagliflozin*}

- What is new or different about this medicine?

This is the third medicine in the class of sodium-glucose co-transporter 2 (SGLT2) inhibitors. It is indicated for the treatment of adults with type 2 diabetes mellitus (T2DM). It has been approved since 2014.

- Is there good quality evidence that it is more effective than existing medication? Empagliflozin is an add-on medicine to be used in combination with existing medicines plus diet and lifestyle modifications for T2DM when a patient's glycated haemoglobin (HbA1c) has not been optimally reduced. Randomised controlled trial (RCT) data suggests that empagliflozin is beneficial in combination with metformin or glimepiride. It also appears to reduce weight and systolic blood pressure compared to placebo. More recently, post-marketing surveillance has led to a new indication for empagiflozin: to reduce the risk of cardiovascular death in adult patients with T2DM and cardiovascular disease. The three pharmaceutical companies that developed an SGLT2 have performed meta-analyses of outcomes. The early studies showed no differences between the three medicines in terms of cost or effectiveness.

- Is the evidence based on RCTs with sufficient participants? The overall effects are based on a systematic review of 10 studies involving 6203 people (as of 2014). The post-marketing surveillance involved a clinical trial of more than 7000 patients.

- Are the studies relevant to my patient/population?

Most people in the trials had had T2DM for less than five years, had a body mass index (BMI) of $25-34 \mathrm{~kg} / \mathrm{m}^{2}$ and the mean age was $50-60$ years.

- Are there any longer term studies with patient-relevant outcomes?

Primary outcome of trials is reduction in HbA1c.

- What are the incidence and severity of side effects?

The most common side effects are urinary tract infections (UTIs) and female genital infections. Empagliflozin can also cause dehydration, hypotension and ketoacidosis. Hypoglycaemia is more common when used with a sulphonylurea or insulin. All three SGLT2s led to increases in all types of cholesterol. In 2016, reports from the US suggested that canagliflozin and dapagliflozin are associated with several cases of leg and toe amputations.

- Are long-term safety data available?

The long-term effects over several years are not yet known. Another SGLT2 has been linked with a drop in estimated glomerular filtration rate.

- Which patients are at most risk of side effects? Women: UTIs and genital infections.

- Which patients should not receive this drug? Patients with type 1 diabetes mellitus (T1DM) or severe kidney problems.

- Do the benefits from this drug outweigh the risks involved? Overall for poorly responding T2DM, SGLT2 agents appear to have benefits for patients.

- Are there some patients who may particularly benefit from this medicine? Patients with raised $\mathrm{HbA1c}$ not lowering sufficiently with other medicines.

- Is it listed on the Pharmaceutical Benefits Scheme (PBS) and, if so, what is the indication? Empagliflozin is PBS-listed for use with other medicines.

- Is it acceptable to patients?

Trial data suggest it is acceptable to patients and well tolerated.

- Monitoring is required in case of adverse events.

*Data drawn from appropriate sources listed in Box 3

media, interactions with peers, specialists and opinion leaders, pharmaceutical advertisements and representatives, ${ }^{18}$ personal experiences, training and, of course, patients. Mindlines need to be created for new medicines, and the process of experimentation is more apparent. GPs need new scripts for discussing a new medicine; they need to be able to interpret the evidence they have read and translate it into appropriate language for individual patients.

Essential information patients need to know about medicines is: what it is for, what it does, potential side effects, how to take it, and do's and don'ts, ${ }^{19}$ including when the medicine should be stopped. Consumers of medicines are encouraged to ask five specific questions to help their decision making (Box 4). ${ }^{20}$ This information may be included in the consumer medicines information (CMI) leaflets written by the pharmaceutical company responsible for the medicine. Some products may not have a CMI; if they do, it should be available either with the packaging or on request when the medicine is dispensed. ${ }^{21}$ However, the prescriber should also interpret and explain this information to patients at the appropriate level of their health literacy. While not all patients will ask such questions, GPs can be proactive in anticipating these queries to help enhance the possibility of a medicine being taken as advised.

We may assume that the majority of patients GPs see regularly trust their doctor to offer them effective choices based on up-to-date knowledge and experience. How people make decisions about taking medicines is complex and includes, but is not limited to, their own or others' experiences and expectations of treatment, media reporting, health beliefs and cost. ${ }^{22}$ Should a GP advise a patient that this is the first time they have prescribed this medicine? It is important to admit what one does not know about a medicine and not be embarrassed about looking up details in front of the patient.

Interpretation of medicines information includes putting known risks into perspective. Research has 
explored people's understanding of and preferences for risk communication. ${ }^{23}$ Factors that influence patients' responses to information about the potential risks of a new medicine include: ${ }^{24}$

- the extent to which they trust the source of the information

- how the medicine might affect their everyday lives

- the potential risks of the medicine in relation to other perceived risks

- previous experiences

- the difficulty and importance of the choice they are making.

Risk needs to be framed for the patient's understanding, avoiding terminology such as 'likely' and 'rarely', which are context-specific. A useful resource on communicating benefits and risks has been developed by the Australian Commission on Safety and Quality in Health Care. ${ }^{25}$

\section{Conclusion}

GPs as prescribers have a responsibility to evaluate new medicines in relation to their possible efficacy and safety profile for individual patients. There are a number of resources that provide accurate and unbiased summaries of information about medicines to help decision making in partnership with patients.

\section{Key points}

- Before prescribing a new medicine, GPs should appraise its suitability for an individual patient by taking into account past history, current medication, allergies and preferences.

- A number of resources provide accurate summaries of the available evidence on new medicines.

- New medicines may have limited evidence in terms of effectiveness and patient safety, as RCTs undertaken during their development have a limited range of participants.

- Only as a medicine is used more widely will rarer adverse events become more obvious.

- GPs need to be able to interpret the evidence relating to new medicines for their patients to enable shared decision making.

\section{Box 3. Sources of independent information about medicines}

- AusDI (www.ausdi.com.au): medicine monographs, company product information and consumer medicines information (CMI), drug-drug interactions, medicine use in pregnancy and breastfeeding, product images. Published by MedicalDirector; updated on the first of every month

- Australian Medicines Handbook (www.amh.net.au): national formulary of medicines including individual medicine monographs and comparative information, drug-drug interactions, medicine use in pregnancy and breastfeeding. Published by Australasian Society of Clinical and Experimental Pharmacologists and Toxicologists, Pharmaceutical Society of Australia and The Royal Australian College of General Practitioners; updated every six months.

- Australian Prescriber (www.australianprescriber.com) considers the merits of drugs recently made available in Australia, and reviews primary literature and the Therapeutic Goods Administration (TGA), US Food and Drug Administration (FDA) and the European Medicines Agency (EMA) regulatory decisions. Published by NPS MedicineWise

- The Cochrane Collaboration (www.cochrane.org) provides systematic reviews about health care interventions.

- MIMS (www.mims.com.au): company product information and CMI, drug-drug interactions, medicine use in pregnancy and breastfeeding, product images; based on the PI. Published by MIMS Australia - used in many general practice software systems; updated on the first of every month.

- NPS MedicineWise website (www.nps.org.au) and Medicine Finder (www.nps.org.au/ medical-info/medicine-finder): provides access to consumer medicine information and other information about medicines.

- NPS RADAR (Rational Assessment of New Drugs and Research) (www.npsradar.org.au): updates on new medicines as they are listed on the PBS; explains Pharmaceutical Benefits Advisory Committee (PBAC) decisions; and focuses on the place in therapy, compared with existing options. Published by NPS MedicineWise

- Therapeutic Guidelines (www.tg.org.au): condition-based clinical practice guideline, along with summary medicines information. Published by Therapeutic Guidelines Limited

- TGA publishes the Australian Public Assessment Reports (AusPARs) free online: includes the clinical evaluation of new medicines by the TGA and product information (www.tga.gov. $\mathrm{au} /$ industry/pm-auspar.htm)

\section{Authors}

Jill Thistlethwaite MBBS, PhD, MMEd, FRCGP, FRACGP, Medical Adviser, NPS MedicineWise, Sydney, NSW; Adjunct Professor, University of Technology Sydney, NSW; Honorary Professor, University of Queensland, QId. jthistlethwaite@ nps.org.au

Lynn Weeks AO, PhD, CEO NPS MedicineWise, Sydney, NSW; Adjunct Professor, University of Queensland, Qld

Competing interests: None.

Provenance and peer review: Commissioned, externally peer reviewed.

\section{Acknowledgements}

We would like to acknowledge Dr Jonathan Dartnell and Dr Clare Weston of NPS MedicineWise for their help in preparing this paper.

\section{References}

1. The Australian Medicines Industry. Medicines milestones: A decade of persistence, prevention and promise. Canberra: The Australian Medicines Industry, 2012. Available at: https:// medicinesaustralia.com.au/wp-content/uploads/ sites/52/2010/01/Medicines-Milestones.pdf [Accessed 18 December 2017].

2. Therapeutic Goods Administration. Prescription medicines and biologicals: TGA annual summary 2016. Canberra: TGA, 2016.

\section{Box 4. Five questions for patients to ask their doctors ${ }^{20}$}

1. Do I really need this treatment?

2. What are the risks?

3. Are there simpler, safer options?

4. What happens if I don't do anything?

5. What are the costs?

3. Martin J, Shenfield G. The hazards of rapid approval of drugs. Aust Presc 2016;39(1):2-3. doi: 10.18773/austprescr.2016.005

4. Abott Laboratories. Sibutramine (brand name Reductil) Information - Australia. North Ryde, NSW: Abott Laboratories, 2010.

5. Therapeutic Goods Administration. Who we are and what we do. Canberra: TGA 2018. Available at www.tga.gov.au/who-we-are-what-we-do [Accessed 18 December 2017].

6. Department of Health. The Pharmaceutical Benefits Scheme. Canberra: DoH, updated 2018. Available at www.pbs.gov.au/info/about-the-pbs [Accessed 18 December 2017]. 
7. Medical Board of Australia. Good medical practice: A code of conduct for doctors in Australia. Canberra: Medical Board of Australia 2014. Available at www.medicalboard.gov.au/ Codes-Guidelines-Policies/Code-of-conduct.aspx [Accessed 18 December 2017].

8. Chen R, Desau NR, Ross JS, et al. Publication and reporting of clinical trial results: Cross sectional analysis across academic medical centers. BMJ 2016;352:i637. doi: 10.1136/bmj.i637.

9. Consolidated Standards of Reporting Trials (CONSORT). CONSORT: Transparent reporting of trials. Ottawa, ON: CONSORT, 2018. Available at www.consort-statement.org [Accessed 18 December 2017].

10. Williams K, Sansoni J, Morris D, Grootenaat P, Thompson D. Patient-reported outcome measures: Literature review. Sydney: Australian Commission on Safety and Quality in Health Care, 2016.

11. National Prescribing Service. Questions to ask when evaluating a new drug. Sydney: National Prescribing Service Limited, 2002. Available at www.nswtag.org.au/wp-content/ uploads/2017/08/nps-questions-to-ask.pdf [Accessed 7 May 2018].

12. Day RO, Snowden L. Where to find information about drugs. Aust Presc 2016;39(3):88-95. doi: 10.18773/austprescr.2016.023.

13. Mason A. New medicines in primary care: $A$ review of the influences on general practitioner prescribing. J Clin Pharm Ther 2008;33(1):1-10. doi: 10.1111/j.1365-2710.2008.00875.x.

14. World Health Organization. Safety of medicines: A guide to detecting and reporting adverse drug reactions. Geneva: WHO, 2002. Available at www.whqlibdoc.who.int/hq/2002/WHO_EDM_ QSM_2002.2.pdf [Accessed 18 December 2017].

15. Therapeutic Goods Administration. Blue card adverse reaction reporting form. Symonston, ACT: TGA, 2015. Available at www.tga.gov.au/ form/blue-card-adverse-reaction-reporting-form [Accessed 18 December 2017].
16. Therapeutic Goods Administration. Medicines safety. Symonston, ACT: TGA, 2018. Available at www.tga.gov.au/medicines-safety [Accessed 18 December 2017].

17. Gabbay J, le May A. Evidence based guidelines or collectively constructed 'mindlines'? Ethnographic study of knowledge management in primary care. BMJ 2004;329(7473):1013.

18. Lublóy Á. Factors affecting the uptake of new medicines: A systematic literature review. BMC Health Serv Res 2014;14:469. doi: 10.1186/1472 6963-14-469.

19. Dickinson D, Rayner DKT. What information do patients need about medicines? Ask the patients They may want to know more than you think. BMJ 2003;327(7419):861.

20. Choosing Wisely Australia. 5 questions to ask your doctor or other healthcare provider. Strawberry Hills, NSW: NPS MedicineWise, 2018. Available at www.choosingwisely.org.au/resources/ consumers/5-questions-to-ask-your-doctor [Accessed 18 December 2017].

21. Therapeutic Goods Administration. Consumer medicines information (CMI). Canberra: TGA, 2014. Available at www.tga.gov.au/consumer medicines-information-cmi\#aboutcm [Accessed 18 December 2017].

22. Jing J, Sklar GE, Oh VMS, Li SC. Factors affecting therapeutic compliance: A review from the patient's perspective. Ther Clin Risk Manag 2008;4(1):269-86

23. Edwards A, Trevena L. What you need to know as a clinician about risk communication. In: Elwyn G, Edwards A, Thompson R, editors. Shared decisionmaking in health care: Achieving evidence-based patient care. 3rd edn. Oxford: Oxford University Press, 2016; p. 112-22.

24. Alaszewski A, Horlick-Jones T. How can doctors communicate information about risk effectively? BMJ 2003;327(7417):728-31. doi: 10.1136/ bmj.327.7417.728
25. Australian Commission on Safety and Quality in Health Care. Helping patients make informed decisions: Communicating risks and benefits. Activity modules 1-4. Sydney: ACSQHC, date unknown. Available at http://contenttest. learningseat.com/safetyandquality/index.html [Accessed 18 December 2017]. 